\title{
Eigenbluttherapie bei Hauterkrankungen
}

Mit einer Eigenblutbehandlung können Sie Ihren Patienten bei vielen Erkrankungen mit einem müde gewordenen Immunsystem wieder auf die Sprünge helfen. Gerade bei Hauterkrankungen ist sie eine häufig angewandte Therapiemöglichkeit. HP Karl Vetter zeigt Ihnen, welche Anwendungsformen der Eigenblutbehandlung sich bei Hauterkrankungen besonders eignen. Viele Tipps und ein Behandlungsbeispiel veranschaulichen eine erfolgreiche und lang bewährte Therapiemethode.

Die Eigenblutbehandlung gehört heute zur alltäglichen Therapieform zahlreicher Heilpraktiker. Sie eignet sich hervorragend zur Behandlung von Krankheiten, bei denen eine Störung des Immunsystems vorliegt. Besonders bei Hauterkrankungen hat sie sich gut bewährt. Insbesondere therapieresistente chronische Ekzeme lassen sich mit Eigenblut gut beeinflussen, aber auch akute Ekzeme können sich bereits nach wenigen Behandlungen wesentlich verbessern. Zu den Hauterkrankungen, die auf eine Eigenbluttherapie gut ansprechen gehören:

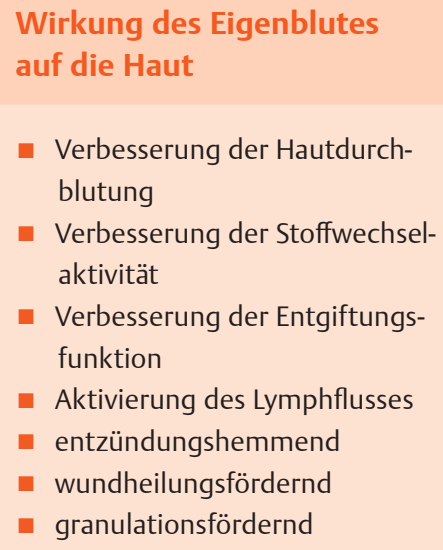

- Verbesserung der Entgiftungsfunktion

- Aktivierung des Lymphflusses

- entzündungshemmend

- wundheilungsfördernd

- granulationsfördernd

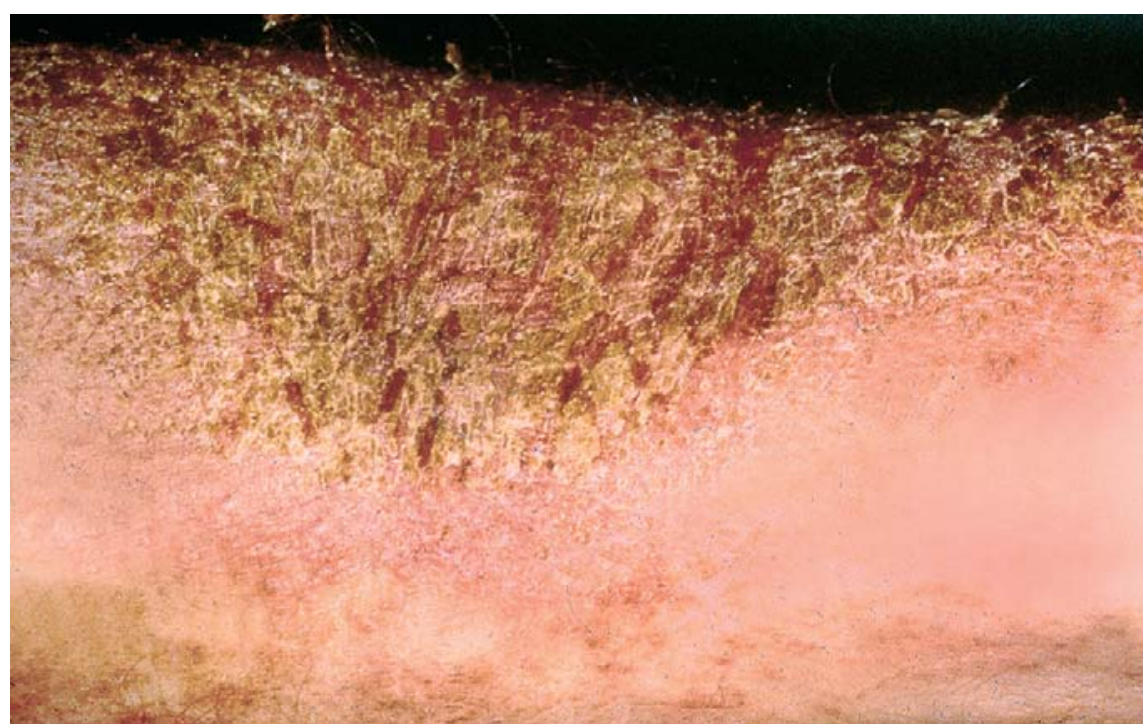

Abb. 1 Neurodermitis: Teigig-entzündlich geschwollene Haut mit Parakeratose. Foto: @ SciencePictures/KES/Thieme
- Neurodermitis
- Akne vulgaris
- Furunkulose
- Urtikaria
- Ekzeme
- Mykosen
- Schuppenflechten
- Herpes simplex/zoster
- chronische Wunden

\section{Wie wirkt die Eigenblut- behandlung?}

Die Eigenblutbehandlung ist eine sogenannte unspezifische Reiztherapie, die das körpereigene Immunsystem anregt und unterstützt. Es wird Blut entnommen und anschließend als eine Art Fremdkörper beispielsweise intramuskulär wieder injiziert. Somit wird das Blut selbst zum Reiz für den Körper und löst eine Aktivierung der körpereigenen Abwehr und Mobilisierung der Selbstheilungskräfte aus.

Die Folge ist eine allgemeine Verbesserung des Befindens und eine positive Beeinflussung gestörter Regulationsvorgänge
(- Kasten). Die Fähigkeit des Organismus, sich mit Krankheiten auseinanderzusetzen, wird deutlich verbessert. Durch die Häufigkeit und Dauer der Behandlung sowie durch die Wahl eines geeigneten Zusatzmittels kann ein spezielles individuelles Wirkungsspektrum erzielt werden.

Die Nebenwirkungen der Eigenbluttherapie sind äußerst gering und resultieren aus deren Wirkung. Die körpereigene Abwehr beginnt, sich mit Dingen auseinanderzusetzen, die bisher nicht aufgearbeitet werden konnten.

Dies kann gelegentlich zu einer
vorübergehenden Erstreaktion oder auch zu einer Erstverschlimmerung der eigentlich behandelten Symptome führen.

\section{Mo̊gliche Anwendungsformen der Eigenbluttherapie}

Je nach Krankheitsbild haben sich in der Praxis verschiedene Anwendungsformen bewährt: 
- intramuskuläre Injektion

subkutane Injektion

homöopathisch potenzierte Tropfen

- Ozon-Sauerstofftherapie (Kleine oder

Große Eigenblutbehandlung)

Bei Hauterkrankungen ist die intramusku-

läre Injektion die häufigste Anwendung.

\section{Intramuskuläre und subkutane Injektion}

Am geläufigsten ist die intramuskuläre bzw. subkutane Injektion von Eigenblut. Hierzu wird eine geringe Menge Blut aus der Ellenbogenvene entnommen und anschließend sofort in den Gesäßmuskel bzw. in das subkutane Fettgewebe gespritzt.

Nach Bedarf kann das Blut mit verschiedenen biologischen Medikamenten zur Wirkungsverstärkung angereichert werden. Meist wird eine Spritzenserie verabreicht, z. B. 10 Behandlungen über 5 Wochen verteilt, wobei der Eigenblutanteil mit jeder Injektion erhöht werden kann. Das Behandlungs-Schema könnte so aussehen:

1. Sitzung: $0,5 \mathrm{ml}$ Blut

2. Sitzung: $1,0 \mathrm{ml}$ Blut

3. Sitzung: 1,5 ml Blut usw. bis $3 \mathrm{ml}$ und dann der gleiche Weg wieder zurück bis $0,5 \mathrm{ml}$ Blut)

\section{Potenzierte Eigenblutbehandlung}

Eine weitere Möglichkeit besteht in der Behandlung mit potenziertem Eigenblut. Dieses kommt dem Prinzip der Homöopathie am nächsten.

Aus einem Tropfen Blut werden nach homöopathischer Potenzierung Eigenbluttropfen hergestellt. Diese enthalten alle Informationen über die vorliegende Krankheit.

Nach den Regeln der Homöopathie wählt man für akute Erkrankungen niedrige und für chronische Krankheiten eher höhere Potenzen. Gerade für Kinder ist die potenzierte Eigenbluttherapie besonders geeignet, da sie keine Angst vor Spritzen überwinden müssen. Kinder sprechen besonders gut auf eine homöopathische Behandlung an, weil meist noch wenige Regulationsblockaden vorliegen. Ich verwende gerne die Potenzen C 3, C 6 und C 9.

\footnotetext{
1 Gemäß AMG darf der Heilpraktiker ein selbst hergestelltes Arzneimittel (z. B. homöopathische Potenzierung der Eigenbluttropfen) dem Patienten nur in der Praxis verabreichen und keinesfalls mitgeben!
}

\section{Eigenblutbehandlungen mit Ozon}

Ozon ist meist nur als Umweltgift in aller Munde, obwohl seine spezielle Bakterien abtötende und Virus inaktivierende Wirkung schon seit mehr als 100 Jahren genutzt wird, z. B. bei der Trinkwasser-Aufbereitung. Medizinisches Ozon ist eine Mischung aus ca. 5 \% Ozon und ca. 95 \% reinem Sauerstoff. Es regt den Zellstoffwechsel an und verbessert dadurch die Entgiftung. Ebenfalls aktiviert es die roten und weißen Blutkörperchen, sodass der Sauerstofftransport erhöht und die Immunabwehr gesteigert wird. Diese Eigenschaft hat eine besonders positive Wirkung auf die Haut und unterstützt daher die Heilungsvorgänge bei vielen Hauterkrankungen.

\section{Große Ozon-Eigenblutbehandlung}

Bei der Großen Ozon-Eigenblutbehandlung werden ca. 50-80 ml Blut aus einer Armvene entnommen und in eine Vakuumflasche geleitet. Durch Zugabe von $10 \mathrm{ml} \mathrm{Na-}$ triumcitrat (speziell für die Ozontherapie) wird das Blut ungerinnbar gemacht. Anschließend versetzt man es mit Ozon-Sauerstoff und vermengt (oxyveniert) es ca. 30-60 Sekunden durch Kreisbewegungen. Danach erfolgt die Reinfusion durch die noch liegende Kanüle zurück in die Vene. Die gesamte Behandlung dauert ca. 15-20 Minuten.

\section{Kleine Ozon-Eigenblutbehandlung}

Hierbei wird das aus der Armvene entnommene Blut ( $2 \mathrm{ml}$ ) in der Spritze durch kräftiges Schütteln mit Ozon-Sauerstoff vermischt. Anschließend spritzen Sie das aktivierte Blut in den Gesäßmuskel (Musculus gluteus maximus) zurück. Dies geschieht in der Regel mit einer ansteigenden Dosierung.

\section{Unterschiedliche Mengen - Unterschiedliche Wirkungen}

Der Heilpraktiker entscheidet je nach Krankheitsbild, wie viel Eigenblut er dem Patienten entnimmt. Als Faustregel könnte die Arndt-Schulz'sche Regel gelten:

- schwache Reize fördern $(0,2-1 \mathrm{ml})$

- mittelstarke Reize hemmen (1,5-3 ml)

- starke Reize lähmen (3,5-5 ml)

Mirjam L.: Ekzem an beiden Oberschenkeln

Die Patientin Mirjam L. ist 31 Jahre, verheiratet, hat 2 Kinder und leidet seit 8 Jahren
Kleine Eigenbluttherapie: Kontraindikationen

v akute Leberentzündung oder andere schwere Leber- und Nierenerkrankungen

- Fieber unbekannter Ursache

- Thrombose, Thrombophlebitis

n bei antikoagulativen Therapien (z. B. Marcumar), etwa nach einem Herzinfarkt

an einem atopischen Ekzem (Neurodermitis) an beiden Oberschenkeln. Mehrere Behandlungen mit diversen Salben einschließlich Kortison brachten bisher keine bzw. nur vorübergehende Wirkung. Mirjam L. ist Erzieherin und fühlt sich immer sehr gestresst. Sie verzehrte viele Süßigkeiten und ernährte sich wie sie selbst sagte, mit „Hausmannskost“.

Bei der durchgeführten Computer-Regulations-Thermographie nach Rost zeigten sich folgende Befunde:

v chronische Kieferhölenherde

- Lymphabflussstörung im Halsbereich

- seitenungleiche Regulation der

Schilddrüse

- Leberbelastung

- Darmdysbiose im Sinne einer

Gährungsdyspepsie

vegetative Hyperregulation

Ich führte über 3 Wochen eine Basistherapie durch: Entgiftung des Körpers mit Metabiarex (Fa. Meta-Fackler Arzneimittel),

Durchführung der Eigenbluttherapie

Ich gebe $18 \mathrm{ml}$ Ozon-Sauerstoff in einer Dosierung von $100 \mu$ in eine $20 \mathrm{ml}$ Spritze. Anschließend entnehme ich mit dieser Spritze $2 \mathrm{ml}$ Blut aus der Vene der Patientin, vermische das Blut durch kräftiges Schütteln und reinjiziere sogleich i.m.

Bei jeder weiteren Behandlung steigere ich die Ozon-SauerstoffMenge bis zu einer Gasamtdosis von $600 \mu$. Die Blutmenge bleibt bei $2 \mathrm{ml}$. 
Metasolidago (Fa. Meta-Fackler Arzneimittel) und Lymphdiaral Basistropfen (Fa. Pascoe), jeweils $4 \times 15$ Tr. tägl., Darmsanierung mit Mutaflor (Fa. Ardeypharm) 3 Tage morgens 1 Kps., ab 4. Tag morgens 2 Kps., Ernährungsberatung (Reduzierung der Süßigkeiten, Umstellung auf eine vollwertige Ernährung, > 21 Flüssigkeitszufuhr - z. B. Wasser, Tee - am Tag).

Danach folgten 10 Behandlungen mit $\mathrm{O}_{3}-\mathrm{O}_{2}$ Eigenblut (- Kasten, S. 32) und Hautfunktionstropfen $4 \times 15 \mathrm{Tr}$. tägl. (Fa. Chosmochema).

Ich habe mich für die kleine Eigenbluttherapie entschieden, weil ich bei Ekzemerkrankungen mit der Ozon-Eigenblutbehandlung die besten Erfahrungen gemacht habe. Ich denke, dass Eigenbluttherapien mit homöopathischen oder phytotherapeutischen Mitteln möglicherweise ähnliche Ergebnisse bringen würden.

Die Eigenbluttherapie mit $\mathrm{O}_{3}-\mathrm{O}_{2}$ habe ich $2 \times$ wöchentlich in einer Dosierung von Anfangs $100 \mathrm{\mu} \mathrm{O}_{3}-\mathrm{O}_{2}$ durchgeführt. Diese Dosis wurde langsam auf $600 \mu \mathrm{O}_{3}-\mathrm{O}_{2}$ gesteigert. Nach der Therapie (5 Wochen) waren die Ekzeme abgeheilt. Die ersten
Besserungsanzeichen stellten sich jedoch schon nach 2 Wochen ein, sodass die Rötung deutlich zurückging.

\section{(1) Weiterführende Literatur}

Krebs H: Praxis der Eigenbluttherapie. München: Elsevier; 2007.

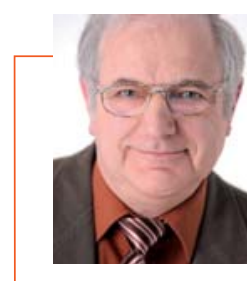

Karl Vetter

Wagnerstr. 10

76709 Kronau

Karl Vetter ist ausgebildeter Krankenpfleger und seit 1992 als Lehrer für Krankenpflege tätig. 1986-1988 Ausbildung zum Heilpraktiker; seitdem führt er eine eigene Praxis in Kronau. 1996 Eröffnung der Kraichgau-Heilpraktikerschule in Sinsheim (Heidelberg). Karl Vetter ist Mitglied im $\mathrm{BDH}$, bei der $\mathrm{HPGO}_{3}$-Gesellschaft sowie Mitglied bei der Deutschen Gesellschaft für Thermographie und Regulationsmedizin.

E-Mail: Karl.Vetter@t-online.de 